\title{
Dependence of the substrate structure and the film growth at the junction of YBCO SEJ rf-SQUIDs on the IBE process and effects on the SQUID's characteristics
}

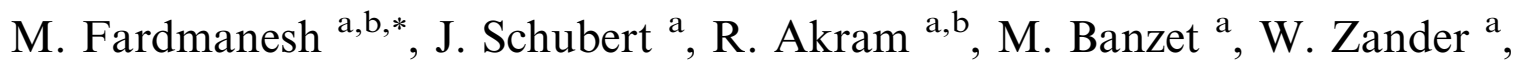 \\ Y. Zhang ${ }^{\text {a }}$, M. Schilling ${ }^{\text {c }}$, H.-J. Krause ${ }^{\text {a }}$ \\ ${ }^{a}$ Institut für Schichten und Grenzflächen, Research Center Juelich, Forschungszentrum Jülich (FZJ) GmbH, D52425 Juelich, Germany \\ ${ }^{\mathrm{b}}$ Department of Electrical and Electronics Engineering, Bilkent University, 065332 Ankara, Turkey \\ ${ }^{\mathrm{c}}$ Institute for Applied Physics (IAP), University Hamburg, 20355 Hamburg, Germany
}

\begin{abstract}
Step edge junction (SEJ) rf-SQUIDs were made of $200 \mathrm{~nm}$ thick YBCO films on $\mathrm{LaAlO}_{3}\left(\begin{array}{ll}1 & 0\end{array}\right)$ substrates using pulsed laser deposition technique. The steps on the substrates were developed using a combination of stationary and rotating angled argon ion beams with different beam energies and intensities. While sharp clean steps with heights up to $300 \mathrm{~nm}$ were obtained on the substrates using the combinatorial ion beam etching (IBE) process, very shallow ramp-type surfaces were found developing on the bottom of the trench, close to the steps. The ramp-type surfaces were found to be a source of hole-type defects in the films grown at the step edges. High quality films could be obtained on the flat regions away from the steps. Higher defect densities in the films close to the SEJs resulted in devices with higher $1 / f$ noise and wider spread of the junction parameters. The $1 / f$ noise of such devices increased with decreasing temperature. High quality films on sharp clean steps with flat substrate surfaces, developed using optimized combinatorial IBE process, resulted in higher yield of low $1 / f$ noise SQUIDs. The $I_{\mathrm{c}}$ of the junctions and hence the working temperature of the SQUID could also be controlled by the junction width and the step height.
\end{abstract}

(C) 2002 Elsevier Science B.V. All rights reserved.

Keywords: rf-SQUID; YBCO thin film; Step edge Josephson junction; $1 / f$ noise

\section{Introduction}

While the step edge junction (SEJ) structure allows high flexibility in the layout designs for

\footnotetext{
${ }^{*}$ Correspponding author. Address: Institut für Schichten und Grenzflächen, Research Center Juelich, Forschungszentrum Jülich (FZJ) GmbH, D52425 Juelich, Germany. Fax: +492461-612630.

E-mail address: m.farmanesh@fz-juelich.de (M. Fardmanesh).
}

Josephson junction (JJ) based devices such as rfSQUIDs, a better control of the parameters of such grain boundary (GB) junctions remains to be further achieved [1]. This is due to the difficulties in control of the step structure fabricated on the substrates, in particular on $\mathrm{LaAlO}_{3}$, and the growth of high quality film at such steps [1-3]. The critical current $\left(I_{\mathrm{c}}\right)$ of the high- $T_{\mathrm{c}}$ GB JJs is also strongly dependent on the microstructure of the film at the GB, and is very sensitive to both the step structure and the film growth [1]. This makes 
the control of the parameters of the SEJ GBs further challenging. There have been some efforts to obtain the desired step structures using various techniques, mainly using an ion beam etching (IBE) process [1-3]. These efforts have mostly been focused on making SEJ rf-SQUID magnetometers and gradiometers where e.g. the bi-crystal GB substrates partly limit the layout designs due to the extension of the straight line GB across the substrate. The bi-crystal GB junctions also result in much higher $J_{\mathrm{c}} \mathrm{JJs}$, compared to that of SEJs, limiting the fabrication due to the need to submicron width junctions [4-6]. This is due to the need of very low $I_{\mathrm{c}}$ for the $\mathrm{JJ} \mathrm{s}$ in range of microamperes for typical designs of rf-SQUIDs [2,5]. While the SEJs favorably result in very low $J_{\mathrm{c}}$ junctions, their $J_{\mathrm{c}}$ can also be varied by the step structure lowering for sharper and deeper steps $[2,6]$.

Very low $1 / f$ noise SEJ rf-SQUID were obtained through the control of the step structure and the growth of high quality $\mathrm{Y}-\mathrm{Ba}-\mathrm{Cu}-\mathrm{O}$ film at the junctions. The films were deposited by pulsed laser deposition technique using an $\mathrm{KrF}$ excimer laser and patterned using conventional photolithography and IBE techniques [7]. We present results on the control of the $\mathrm{LaAlO}_{3}$ substrate step structure through the IBE process and the effects of the step structure on the film growth at the steps and on the SQUIDs' characteristics. The SQUIDs were made using our typical rf-SQUID magnetometer layout designs with $(100 \mu \mathrm{m})^{2}$ loop and $3.6 \mathrm{~mm}$ diameter washer area [8].

\section{The substrate step structures and the film growth}

\subsection{Ramp-type step structures}

Different ramp-type structures were made onto $\mathrm{LaAlO}_{3}\left(\begin{array}{lll}1 & 0 & 0\end{array}\right)$ substrates using stationary $40-45^{\circ}$ angled argon ion beam perpendicular to the edges of the ditches in the substrates. The steps were made using ion beam energy levels of $250-600 \mathrm{eV}$ and beam intensities of about $0.1-0.5 \mathrm{~mA} / \mathrm{cm}^{2}$. A typical $60 \mathrm{~nm}$ thick e-beam evaporated gold layer was deposited onto the substrates prior to the step etching process to obtain, clean edges at the steps. The ditches were patterned onto the substrates, using modified photolithography process to obtain sharp photo-resist shadow mask patterns.

Two main types of ramped structures were obtained, based on the direction of the incident ion beam with respect to the step edges on the substrates, as shown in Fig. 1. The ramped structures were not sensitive to the ion beam energy or intensity parameters. The ramp-type step structures obtained on $\mathrm{LaAlO}_{3}$ in the direction of the incident ion beam are shown in Fig. 1a and b. Fig. 1c and d shows the steps covered with $200 \mathrm{~nm}$ thick $\mathrm{Y}-\mathrm{Ba}-\mathrm{Cu}-\mathrm{O}$ films. Similar results were also obtained on $\mathrm{SrTiO}_{3}$ substrate material. High quality or defect free films with smooth morphology could not be obtained on the ramp-type surfaces without accepting deteriorated film quality on the flat surfaces away from the steps. The ramps at the steps resulted in high $J_{\mathrm{c}} \mathrm{JJ} \mathrm{s}$, as compared to the sharp steps discussed in the following section. The SEJ rf-SQUIDs fabricated with junctions like the one in Fig. 1 showed high $1 / f$ spectra with very high operating temperatures close to $T_{\mathrm{c}}$ of the films ( $\sim 90 \mathrm{~K})$, when made using our typical rf-SQUID layouts designs with junction widths of $2-5 \mu \mathrm{m}$ [2].

\subsection{Sharp step structures and effects of IBE parameters}

While relatively sharp steps can be made using conventional normal incident IBE process, the normal ion beam is also found to cause high redeposition of presumably the substrate material at the step edges, resulting in very low yield and unstable noisy devices [2,7]. The redeposition is observed to be much higher on $\mathrm{LaAlO}_{3}$ substrates, as compared to $\mathrm{SrTiO}_{3}$ substrates [2,7]. Using a combination of angled rotating and stationary (along the step edges) ion beams, sharp step structures were made onto the substrates, as shown in Fig. 2. The step structures showed high sensitivity to all the parameters of the ion beam, when prepared using angled ion beam along the step edges. This approach could also lead to rough step structures or light redeposition of the substrate material at the steps, when unoptimized IBE parameters were used as for the sample shown in Fig. 2a. A shallow ramp-type surface with a height up to about $20 \%$ of the step heights was 

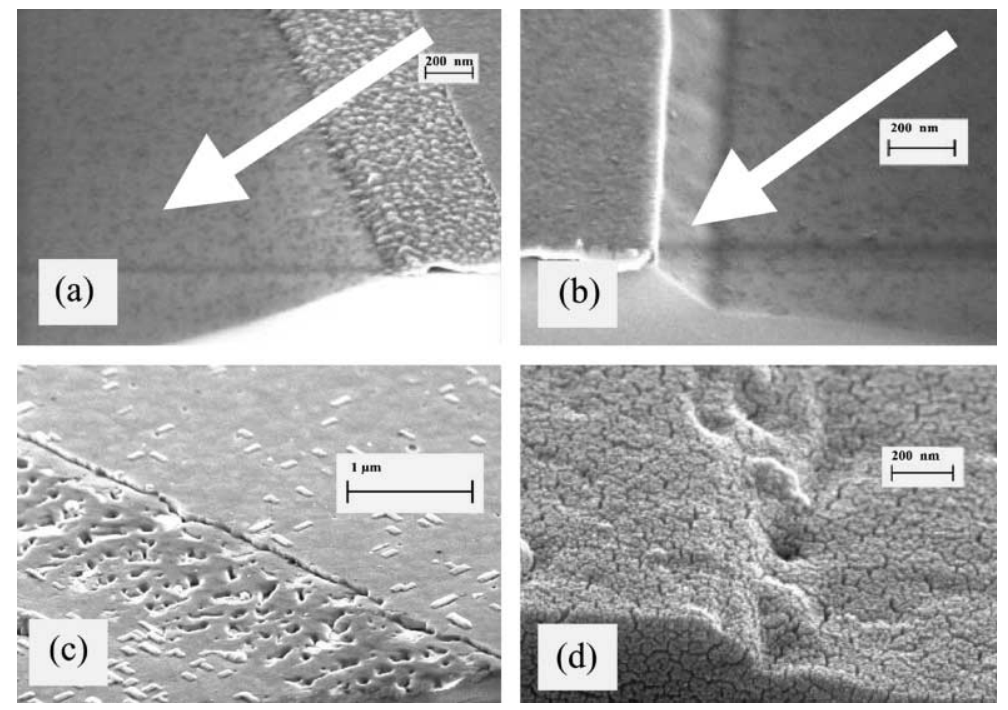

Fig. 1. SEMs of ramp-type step structures on $\mathrm{LaAlO}_{3}$ substrates. (c) and (d) are SEMs of $200 \mathrm{~nm}$ thick $\mathrm{Y}-\mathrm{Ba}-\mathrm{Cu}-\mathrm{O}$ film on the steps in (a) and (b) respectively. The substrates in (a) and (b) and the film in (d) are covered with a thin gold layer ( $<100 \mathrm{~nm})$. Arrows indicate the direction of the ion beam with respect to the step edge.
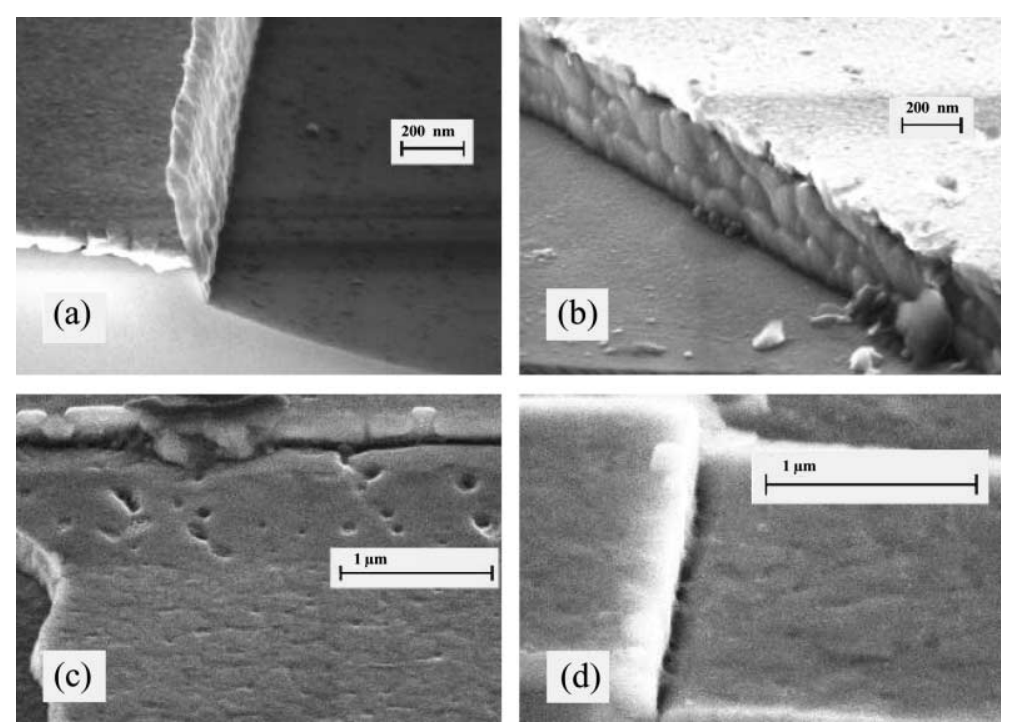

Fig. 2. SEMs of CIBE sharp step structures on $\mathrm{LaAlO}_{3}$ substrate. (c) and (d) are SEMs of $200 \mathrm{~nm}$ thick Y-Ba-Cu-O films deposited on the steps shown in (a) and (b), respectively. The substrates in (c) and (d) are covered with a thin gold layer.

also observed to form at the bottom of the steps in this IBE process. The ramped surfaces resulted in defected film on the bottom of the trench close to the step edge as shown in Fig. 2c.
The height of the ramped surfaces at the steps was found to be dependent on the intensity of the ion beam and the step height, reducing when the beam intensity or the step height was reduced. 
Sharp clean steps with flat surfaces at the steps were obtained on $\mathrm{LaAlO}_{3}$ substrates using an optimized "Combinational IBE" (CIBE) process. This was made using a relatively low intensity $\left(\sim 0.1 \mathrm{~mA} / \mathrm{cm}^{2}\right)$ and high energy $(\sim 600 \mathrm{eV})$ stationary $40^{\circ}$ angled ion beam along the step edges to reach the approximate desired step height, and using a lower energy $(\sim 300 \mathrm{eV})$ rotating $45^{\circ}$ angled ion beam to get surface modified steps shown in Fig. 2b. High quality films in Fig. 2d with smooth morphology at the steps were obtained on $\mathrm{LaAlO}_{3}$ substrates, with steps prepared using the above CIBE process.

\section{CIBE SEJ rf-SQUID's characteristics}

Rf-SQUID magnetometers were made using the CIBE steps on $\mathrm{LaAlO}_{3}$. The CIBE steps favorably provided very low $J_{\mathrm{c}} \mathrm{JJs}$, compared to that of the ramp-type steps. The $J_{\mathrm{c}}$ of the junctions on the CIBE steps showed strong dependence on the step height, with decreasing $J_{\mathrm{c}}$ as the step height increased. The $I_{\mathrm{c}}$ of an array of junctions, made on the CIBE steps, also increased with the increase of the junction widths from $2-8 \mu \mathrm{m}$ on one chip, showing a junction effective area proportional to the geometric junction area [6]. The field dependence of the CIBE SEJ arrays showed very low applied magnetic field sensitivity, as compared to other type of the GB junctions such as bi-crystal GB junctions [6]. The dependence of the $I_{\mathrm{c}}$ of the junctions on the step height and the junction width allowed the control of the operating temperature range of the SQUIDs.

The dependence of the operating temperature range of the SQUIDs on the step structures was investigated by measuring the amplitude of the flux-voltage transfer function signal, $V_{\mathrm{s}-\mathrm{pp}}$, of the devices versus temperature. The $V_{\text {s-pp }}$ of rf-SQUID magnetometers with $2-3 \mu \mathrm{m}$ wide junctions on $\mathrm{LaAlO}_{3}$ substrates with various CIBE step structures is shown in Fig. 3. SQUID 1 with a $275 \mathrm{~nm}$ deep ditch shown in Fig. 3, showed an operating temperature range close to SQUID 2 with a $205 \mathrm{~nm}$ deep ditch. Both devices have $3 \mu \mathrm{m}$ wide junctions. This is interpreted to be due to the ramped surfaces

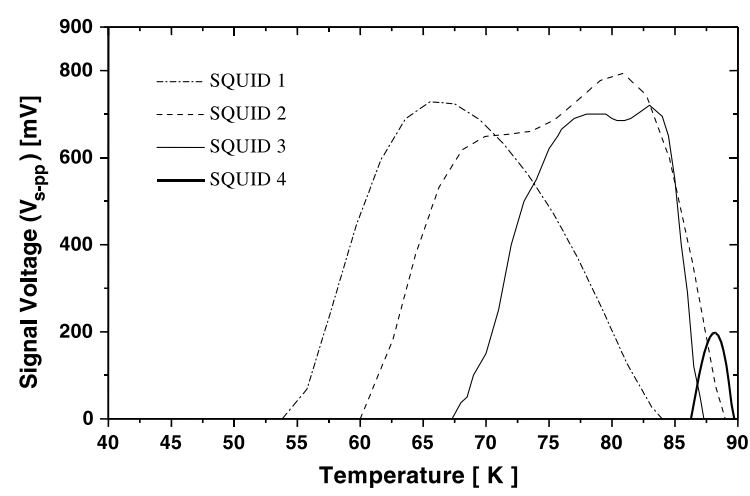

Fig. 3. Flux to voltage transfer function signal, $V_{\text {s-pp }}$, versus temperature of rf-SQUID magnetometers, with $100 \mu \mathrm{m} \times$ $100 \mu \mathrm{m}$ loops. SQUID 2 has a $205 \mathrm{~nm}$ deep ditch and SQUID 3-4 have $135 \mathrm{~nm}$ deep ditches, made using the optimized CIBE process. SQUID 1 has a $275 \mathrm{~nm}$ deep ditch, made using unoptimized CIBE process. The junction width of SQUID 3 is 2 $\mu \mathrm{m}$, and the other devices have $3 \mu \mathrm{m}$ wide junctions.

at the bottom of the ditch, close to the step edges of SQUID 1 made using unoptimized IBE parameters, leading to a step height close to that of SQUID 2. The ramp-type substrate surfaces at the steps of SQUID 1 also led to similar defected film growth as shown in Fig. 2c, leading to a higher $1 / f$ noise of about $40 \mu \Phi_{0} / \sqrt{\mathrm{Hz}}$ at $10 \mathrm{~Hz}$.

Decrease of the step height to $130 \mathrm{~nm}$, using optimized parameters, resulted in a very broad operating temperature range for the SQUIDs, close to the $T_{\mathrm{c}}$ of the $\mathrm{Y}-\mathrm{Ba}-\mathrm{Cu}-\mathrm{O}$ films, as shown for SQUID 4 in Fig. 3. Decrease of the junction width for the same step height reduced the operating temperature range to the desired temperature window arround $77 \mathrm{~K}$. SQUID 3, made on $130 \mathrm{~nm}$ deep CIBE steps with $2 \mu \mathrm{m}$ wide junction, showed as in operating temperature range close to that of SQUID 2, with $205 \mathrm{~nm}$ deep step and $3 \mu \mathrm{m}$ wide junction. The flat surfaces of CIBE step structure of SQUID 3 also led to uniform high quality film inside and outside of the ditch, leading to lower white noise and low $1 / f$ noise. The noise spectra of the sample, measured at liquid nitrogen temperature using conventional $\mathrm{L}-\mathrm{C}$ tank circuit, is shown in Fig. 4. A white noise level of about 15 $\mu \Phi_{0} / \sqrt{\mathrm{Hz}}$ (or $135 \mathrm{fT} / \sqrt{\mathrm{Hz}}$ for the bare SQUID) was measured. 


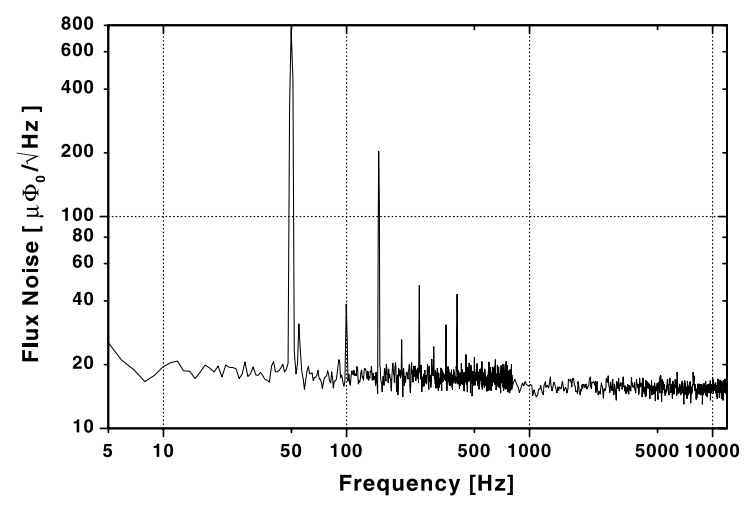

Fig. 4. Noise spectrum of the rf-SQUID magnetometer sample SQUID 3 from Fig. 3 at $77 \mathrm{~K}$ (liquid nitrogen temperature). The field sensitivity of the bare SQUID at the white noise level is $135 \mathrm{fT} / \sqrt{\mathrm{Hz}}$.

\section{Summary and conclusions}

Different type of step structures for step edge GB JJs on $\mathrm{LaAlO}_{3}$ substrate were made using various argon IBE processes. Ramp-type step structures were obtained when stationary angled ion beam perpendicular to the step edges were used. Conventional normal incident ion beam etching resulted in high redeposition of the substrate materials at the step edges, particular on $\mathrm{LaAlO}_{3}$. While the ramp-type step structures resulted in much higher $J_{\mathrm{c}}$ JJs compared to that of the sharp steps, it also resulted in growth of low quality $\mathrm{Y}-\mathrm{Ba}-\mathrm{Cu}-\mathrm{O}$ films with high density of defects on the trenches, when the growth was optimized for high quality films on the $\left(\begin{array}{lll}1 & 0 & 0\end{array}\right)$ flat surfaces of the substrates, away from the steps. Sharp steps were obtained by using stationary angled ion beam along the edges of the steps. Shallow ramped surfaces were also observed to develop at the bottom of ditches, close to the step edges in this IBE process, leading to defect-rich films close to the step edges when unoptimized IB parameters were used. Sharp clean steps with flat substrate surfaces at the steps were obtained using an optimized combinatorial IBE process, favorably resulting in lower $I_{\mathrm{c}}$ junctions. The junctions parameters are controllable by the junction width and the step height, leading to control of the operating temperature range of rf-SQUIDs made with this kind of GB junction. Devices with very low $1 / f$ noise were obtained on substrates with sharp steps, when using the combinatorial IBE process in combination with high quality $\mathrm{Y}-\mathrm{Ba}-$ $\mathrm{Cu}-\mathrm{O}$ films.

\section{Acknowledgements}

This work has been supported in part by the BMBF and TUBITAK research and development joint project no. 42.6.I3B.2.A.

\section{References}

[1] A.I. Braginski, in: H. Weinstock (Ed.), SQUID Sensors: Fundamentals, Fabrication and Applications, NATO ASI Series, Kluwer Academic, Dordrecht, 1996, p. 235.

[2] M. Fardmanesh, J. Schubert, R. Akram, M. Bick, Y. Zhang, M. Banzet, W. Zander, J. Krause, M. Schilling, IEEE Trans. Appl. Supercond. 11 (2001) 1363.

[3] C.P. Foley, E.E. Mitchell, S.K.H. Lam, B. Sankrithyan, Y.M. Wilson, D.L. Tilbrook, S.J. Morris, IEEE Trans. Appl. Supercond. 9 (1999) 4281.

[4] K. Enpuku, T. Minotani, F. Shiraishi, A. Kandori, S. Kawakami, IEEE Trans. Appl. Supercond. 9 (1999) 3109.

[5] M. Bick, J. Schubert, M. Fardmanesh, G. Panaitov, M. Banzet, W. Zander, Y. Zhang, H.-J. Krause, IEEE Trans. Appl. Supercond. 11 (2001) 1339.

[6] M. Fardmanesh, J. Schubert, R. Akram, M. Bick, M. Banzet, W. Zander, J.-H. Krause, presented at the European Conference on Applied Superconductivity, Copenhagen, Denmark, August 26-30, 2001.

[7] J. Schubert, M. Siegert, M. Fardmanesh, W. Zander, M. Proempers, C. Buchal, J. Lisoni, C.H. Lei, Appl. Surf. Sci. 168 (2000) 208.

[8] Y. Zhang, IEEE Trans. Appl. Supercond. 11 (2001) 1038. 